\title{
SCRIPT: Usability of Hand \& Wrist Tele-Rehabilitation for Stroke Patients Involving Personal Tele-Robotics
}

\author{
Alexander Steffen ${ }^{1}$, Johannes Schäfer ${ }^{1}$, Dr. Farshid Amirabdollahian ${ }^{2}$ \\ ${ }^{1}$ User Interface Design, Germany \\ ${ }^{2}$ University of Hertfordshire, United Kingdom \\ alexander.steffen@uid.com
}

\begin{abstract}
We present the overall user experience designed for supporting rehabilitation of stroke patients at home. Patients use a robotic hand (orthosis) to control therapeutic games and a touch screen for the UI. They are supervised remotely by a therapist who uses a similar interface from their desk. The system includes therapeutic games and user interfaces (UIs) for both patients and therapists. The concept and design of these UIs were implemented during the first year of the SCRIPT project
\end{abstract}

Keywords: Tele-Rehabilitation; Stroke; Usability; Motivation; Gamification; Feedback

\section{Introduction}

Due to the high costs of clinical neuro-rehabilitation, post stroke treatments are generally limited to some weeks to a few months after the (stroke) event, with a restricted amount of training due to therapist/healthcare professionals (HCPs) availability. Recent developments in robotmediated rehabilitation have shown the potential of robotic devices for delivering repetitive training and thus offering more patients a post-stroke training.

Patients need to engage in such a tele-robotic rehabilitation for therapy success. The system must be motivating and accessible as well as fast and easy to use. Thus the user experience (UX) of the system is crucial. For the SCRIPT project such a user interface (UI) for patients and HCPs has been conceived. These UIs provide access to training, feedback and communication between patient and HCP as well as access to the patient's progress (game outcome, sensory data, etc). The HCP can adapt the training plan according to therapeutic progress and goals. Patients can use this tele-rehabilitation platform in their homes. It will be supervised and managed remotely. It is innovative in addressing wrist and hand rehabilitation which has been mostly neglected in research to date.

This paper aims at shortly presenting the status of the ongoing work on the SCRIPT project. It introduces the UIs for the patient and HCPs while giving a more detailed picture about the therapeutic games. We will conclude this paper by mentioning current and future evaluations of the system.

\section{Method}

The SCRIPT project follows a User-centred design (UCD) process (ISO 9241-210), which iteratively pro- gresses through the phases of Analysis, Design, Implementation and Evaluation.

During analysis the project partners integrated their expertise from different backgrounds [1-9] to make sure all aspects are covered.

The design phase iterated quickly from hand-drawn sketches to wireframes and finally high-end visual design. For these quick cycles feedback from clinical and technical partners and end-users was elicited and integrated into a concise UI specification. The following sections describe the elements of the overall user experience for the SCRIPT system in more detail. From the outset motivating elements played an important part during UI design. In early sketches engaging UI elements were conceived together with possibilities for feedback about game performance and therapeutic progress. See figure 1.

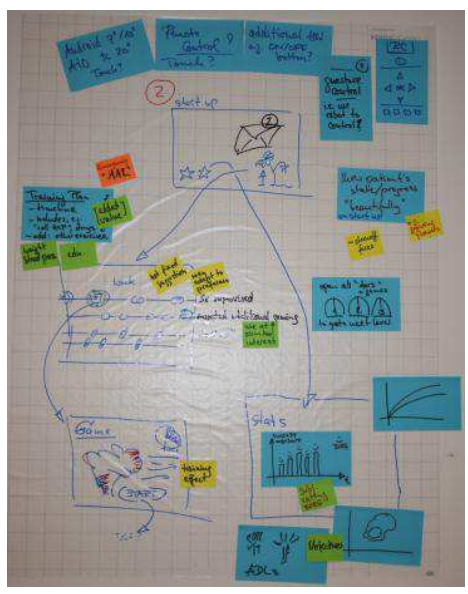

Figure 1: First ideas for the Patient's home screen

\section{User Interface}

The main motivation for the patient is to improve hand and wrist function. This means playing therapeutic games while training their impaired hand. After start-up one game is presented as "Suggested Game". The patient can directly start the game by pressing "Play". The training plan is set up by a therapist and determines which game and level of game is appropriate for each individual. Patients control the UI with the non-impaired hand via a touch screen. The patient wears an individualized robotic hand (orthosis) which is used for game play while training of hand and wrist function. 
Figure 2 shows the visual design for the patient's home screen. The main navigation is placed at the bottom of the screen to accommodate usage independent of the side of the impairment. This interface was kept very simple and straightforward: Patients may be less computer-literate and might also have impairments associated with their stroke, such as visual neglect which can influence their use of screens. Figure 3 gives an impression of the therapist's UI.

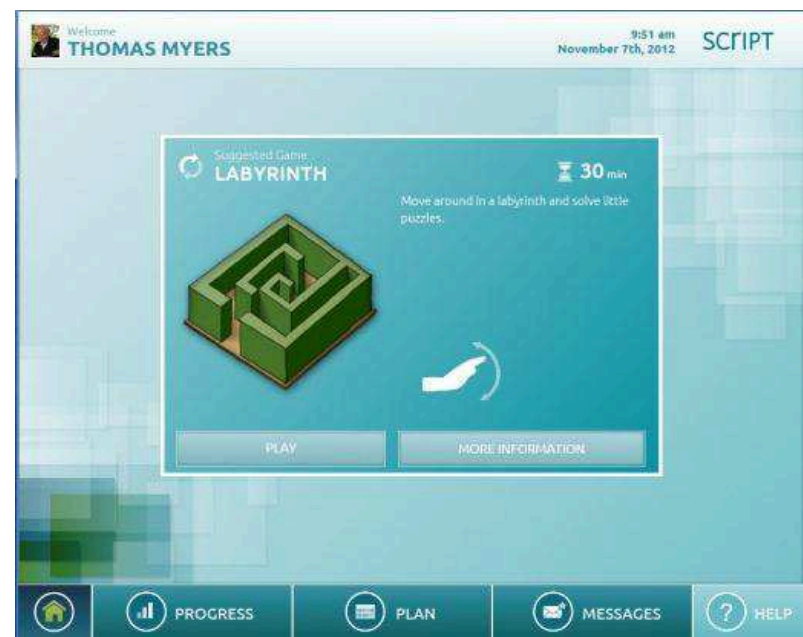

Figure 2: The Patient's home screen

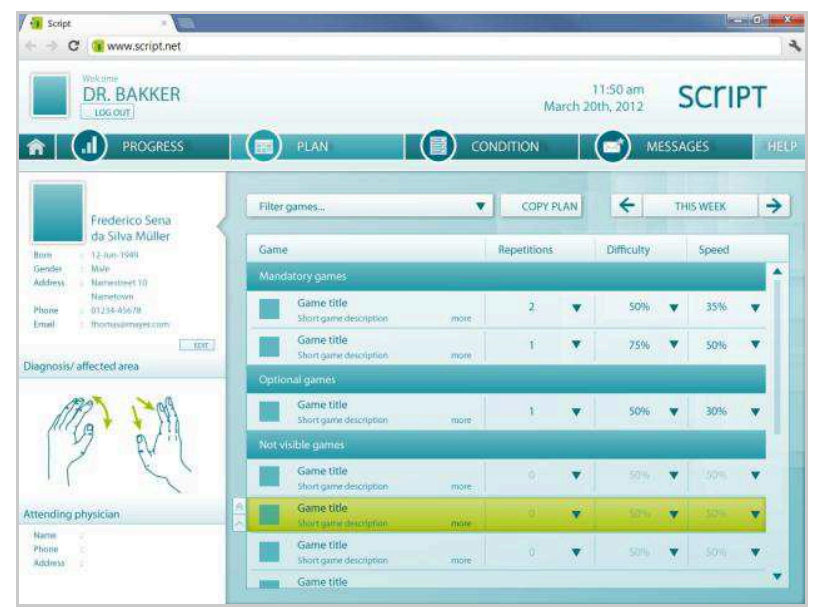

Figure 3: the Therapists's UI

\section{Results}

The SCRIPT project has produced a working platform with a motivating user interface to support stroke patients in rehabilitation at home.

The system has now entered intensive evaluation that will continue in the following year with patients in real-use situations at home. The system will be improved and enhanced in parallel and subdued to further evaluation in the third year.

We believe that this system will be beneficial to patient recovery and for improving overall quality of life and that it may reduce hospital and home visits for patients and caregivers.

\section{Acknowledgement}

SCRIPT (Supervised Care \& Rehabilitation Involving Personal Tele-robotics) is partially funded by the European Commission (FP7-ICT-2011-07; grant 288698). Project co-ordinator: Farshid Amirabdollahian.

\section{Bibliography}

[1] Amirabdollahian, F., R. Loureiro, E. Gradwell, C. Collin, W. Harwin and G. Johnson (2007). "Multivariate analysis of the Fugl-Meyer outcome measures assessing the effectiveness of GENTLE/S robotmediated stroke therapy." J Neuroeng Rehabil 4: 4.

[2] Amirabdollahian, F., R. Loureiro and W. Harwin (2002). "Minimum jerk trajectory control for rehabilitation and haptic applications." 2002 IEEE International Conference on Robotics and Automation, Vols I-IV, Proceedings: 3380-3385.

[3] Cieslik, Silvana; Klein, Peter; Compagna, Diego; Shire, Karen (2012): Das Szenariobasierte Design als Instrument für eine partizipative Technikentwicklung im Pflegedienstleitungssektor. In: Shire, Karen; Leimeister, Jan Marco (Hg.): Technologiegestützte Dienstleistungsinnovation in der Gesundheitswirtschaft. (ISBN: 978-3-8349-3505-2) Wiesbaden: Springer Gabler.

[4] Hermens, H. J. and M. M. R. Vollenbroek-Hutten (2008). "Towards remote monitoring and remotely supervised training."Journal of Electromyography and Kinesiology 18(6): 908-919.

[5] Prange, G. B., M. J. A. Jannink, C. G. M. Groothuis-Oudshoorn, H. J. Hermens and M. J. Ijzerman (2006) "Systematic review of the effect of robot-aided therapy on recovery of the hemiparetic arm after stroke "Journal of Rehabilitation Research and Development 43(2): 171-183.

[6] Prange, G. B., T. Krabben, G. J. Renzenbrink, J. de Boer, H. J. Hermens and M. J. A. Jannink (2009). An explorative study into changes in reach performance after gravity compensation training in chronic stroke patients. In. International Conference on Rehabilitation Robotics (ICORR09), Kyoto, Japan.

[7] Rossini, P. M., C. Calautti, F. Pauri and J. Baron (2003). "Post-stroke plastic reorganization in the adult brain. "The Lancet Neurology 2(8): 493:502.

[8] Stienen, A. H., E. Hekman, G. B. Prange, M. Jannink, F. C. T. Van der Helm and H. Van der Kooij (2009a). "Freebal: design of a minimal weight-support system for upper-extremity rehabilitation." ASME J Med Dev 3: 1-9.

[9] Stienen, A. H. A., E. E. G. Hekman, F. C. T. van der Helm and H. van der Kooij (2009b). "Self-Aligning Exoskeleton Axes through Decoupling of Joint Rotations and Translations." IEEE Transactions on Robotics 25(3): 628-633. 